\title{
Glove Based User Interaction Techniques for Augmented Reality in an Outdoor Environment
}

\author{
B. H. Thomas, W. Piekarski \\ Wearable Computer Laboratory, School of Computer and Information Science, \\ University of South Australia, Mawson Lakes, SA, Australia
}

\begin{abstract}
This paper presents a set of pinch glove-based user interface tools for an outdoor wearable augmented reality computer system. The main form of user interaction is the use of hand and head gestures. We have developed a set of augmented reality information presentation techniques. To support direct manipulation, the following three selection techniques have been implemented: two-handed framing, line of sight and laser beam. A new glove-based text entry mechanism has been developed to support symbolic manipulation. A scenario for a military logistics task is described to illustrate the functionality of this form of interaction.
\end{abstract}

Keywords: Augmented reality; Glove based interaction; User interactions; Wearable computers

\section{Introduction}

We believe a wearable computer with an Augmented Reality (AR) [1] user interface allows for exciting new applications to be deployed in an outdoor environment. We refer to these systems as an Outdoor Wearable Augmented Reality Computer System (OWARCS). Like other researchers, we are taking the use of AR from the indoor setting and placing it in the outdoor environment. There have been a number of systems for outdoor augmented reality such as MARS [2], Touring machine [3], NRL BARS system [4], previous UniSA Tinmith navigation systems [5,6], and UniSA ARQuake [7]

The operation of wearable computers in an outdoor setting is hampered by the lack of suitable input devices. Many traditional input devices such as mice and keyboards are not suitable for mobile work out- doors, as they require a level flat surface to operate. A second difficulty is the well-known registration problem. The field of Virtual Reality (VR) also suffers from the lack of proper input devices and sub-optimal tracking systems, and as a result, new input devices, interfaces, and trackers are continuing to be developed in an attempt to solve these problems. However, many of these devices require fixed infrastructure and are not useable in mobile outdoor environments. Two excellent papers by Azuma [1,8] explain the problems of working outdoors, and the various technologies that are currently available.

The problem of registering virtual images with the user's view of the physical world is a main focus of AR research. However, there is little previous work in the area of user interfaces for controlling AR systems in an outdoor setting, which is one of the focuses of this paper. Two major issues for the development of these 
user interfaces are as follows: firstly, registration errors will make it difficult for a user to point at or select small details in the augmentation and secondly, pointing and selecting at a distance are known problems in virtual and augmented reality applications (compounded by the fact the user is outdoors with less than optimal six degree of freedom tracking of their head and hands).

Therefore, new user interaction techniques are required for an OWARCS, and to state the obvious, the input techniques the users are required to use will have a large impact on the usability of an OWARCS. A key element to the new user interactions is that the augmented reality systems have a varying number of coordinate systems (physical world, augmented world, body relative and screen relative) within which the user must work. In an outdoor application the registration errors of objects at a distance amplify the differences between the physical and augmented world coordinate systems.

The user interface technology presented in this paper has been implemented as part of the Tinmith software system. Only a subset of this technology has been incorporated into working applications at this time. The proposed use of the technology is presented as a scenario. This scenario provides an insight into how we believe such technology may be used to improve user interfaces of OWARCS.

The paper first presents a scenario of using augmented reality to facilitate communication between a number of people in a logistics framework. This scenario presents a proposed collaboration application to highlight how our new interaction techniques may be employed. The issues for developing input mechanisms of an OWARCS are discussed, along with original OWARCS user interface technology, Tinmith-Hand. A number of interaction techniques have been developed to extend Tinmith-Hand to support applications in different domains, such as collaboration. Finally, some implementation details are presented.

\section{Collaboration Scenario}

Collaboration technology facilitates multiple users accomplishing a large group task. There are a number of ways technology may help these users: combine or merge the work of multiple users, prevent and/or inform users when an item of data is being modified by more than one user, and track the activities of multiple users. One major function of collaborative technology is to help people communicate ideas; collaborative electronic whiteboards are a good example of how collaboration technology may help multiple users com- municate, for example the Teamboard system [9]. As with collaborative systems such as distributed white boards and remote video conferencing systems, a main aim of using an OWARCS is to improve communication between the multiple users to attain their common goal. AR's property of overlaying contextually aware information on the physical world is a powerful cueing mechanism to highlight or present relevant information. The ability to view the physical world and augmented virtual information in place between multiple people is the key feature to this form of collaboration technology.

The use of hand-held computing devices communicating via a wireless network has been investigated as a means to facilitate collaboration by Fagrell et al. [10]. Their architecture FieldWise is based on two application domains: first, mobile and distributed service electricians; and second, mobile news journalists. An alternative to hand-held computing, wearable computers leave the hands free when the user is not interacting with computer but still allows the user to view data in the privacy of a Head Mounted Display (HMD). A major research issue is the interaction techniques for users to control and manipulate augmented reality information in the field [11]. We propose the use of augmented reality in the field (outdoors) as a fundamental collaboration tool that may be used across a number of application domains, such as maintenance, military, search and rescue, and GIS visualisation. A number of researchers are investigating augmented reality with wearable computers for distributive collaboration systems [12-15], and our work presented in this paper focuses on direct manipulation user interface issues.

This scenario presents augmented reality user interface tools to support collaboration through enhanced communications channels. Core to making such collaboration feasible is the integration of such communication with existing information systems, such as workflow, logistics, and database systems [16], but this scenario focuses on the mobile user interface issues for distributive collaboration systems incorporating a wide variety of information forms and media. The augmented reality user interface tools presented in this scenario have been developed, but we propose how these tools would be placed in a larger information system to emphasise how such tools could enhance large real world applications. As such, these user interface tools do not communicate with workflow, logistics, and/or database systems, but they are integrated into our mobile AR system Tinmith (Fig. 1).

To understand how a collaborative OWARCS relates to existing collaboration systems, we use the timeplace taxonomy [17]. The time-place taxonomy is 

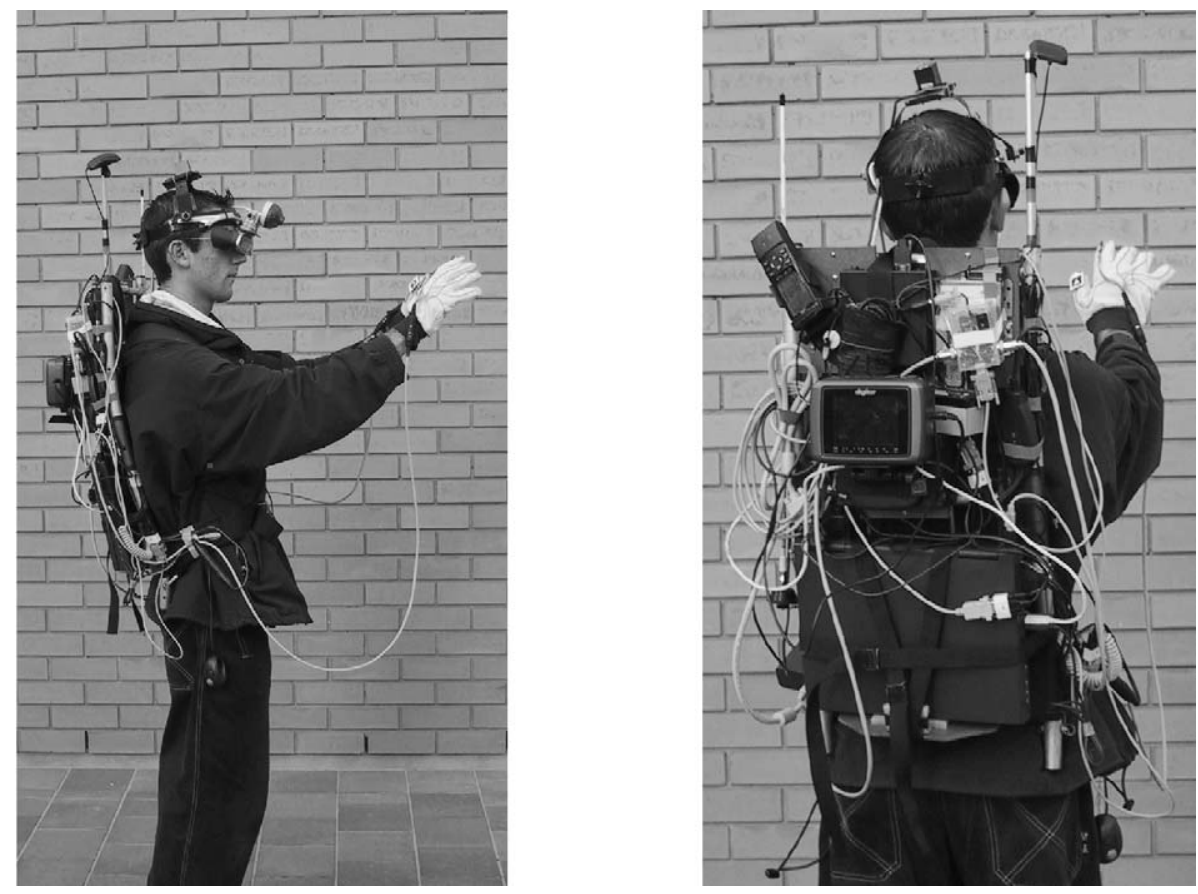

Fig. 1. Outdoor Tinmith backpack computer

defined by the position of the users (same or different) and the time of operation of the collaborative system (same or different). A distinctive quality of activities using a collaboration OWARCS is the ability to use all four time-space configurations, while many existing collaboration systems support activities in one or two configurations. An example of how a collaboration OWARCS would seamlessly cross these four timespace configurations is presented here as a scenario for a logistics task of supporting an overseas military contingent. ${ }^{1}$

The scenario starts with an urgent request from an aviation maintenance person for a replacement rotor for a helicopter and they place a virtual marker on the rotor to have the logistics supervisor contact him. (Figure 2 depicts the geographical placement of the different personnel involved in the process of getting the rotor delivered to the aviation maintenance person.) The location of the rotor in the warehouse is indicated to the warehouse clerk with augmented reality information in the form of virtual signposts and virtual line markings on the floor. The warehouse clerk quickly finds the rotor, and the rotor is moved from the warehouse to the airfield loading dock. The warehouse

\footnotetext{
${ }^{1}$ To make this scenario realistic, we sought advice about military logistics from Dr. Rudi Vernik of the Australian Defence Science and Technology Organisation.
}

clerk attaches an augmented reality information sticker to the rotor's container stating this is an urgent request. This provides a different time-same place configuration for communicating between the warehouse clerk who placed the rotor in the loading dock and the logistics supervisor at the airfield monitoring the shipment of the rotor. The annotation is designed to overcome the problem that the container might be hidden behind other containers. This annotation may be one or more forms of multimedia information, such as text, line drawings, 3D graphics, audio, voice, digital image, or digital video. The annotation is registered to the container containing the rotor. The location of the container can be determined through the use of smart sensors or similar technology. The delivery is also recorded in a standard logistics database for information tracking.

At a later time, the logistics supervisor proceeds to check the supplies to be loaded onto the plane. The logistics supervisor reads the virtual note left on the rotor's container: 'There are a number of different rotors for the different models of helicopters, please contact .... He contacts the aviation maintenance person who placed the original order. This information is shown in their HMD and is retrieved through the identification of the smart badge. The aviation maintenance person asks the logistics supervisor to visually inspect the rotor. The logistics supervisor opens the container and shows the aviation 


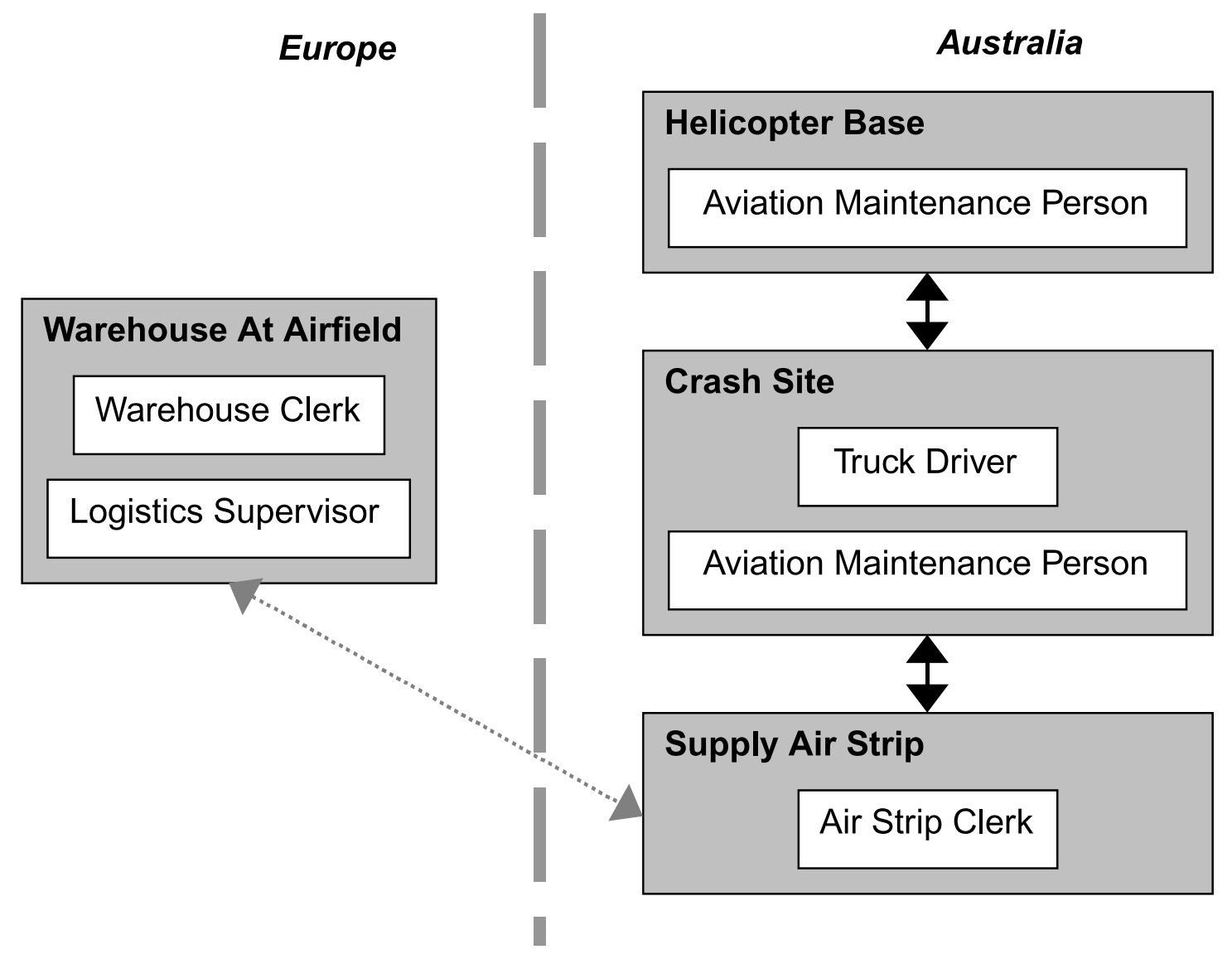

Fig. 2. Location of the different players in the transfer of the rotor.

maintenance person the rotor via a digital camera mounted on their helmet. This situation is now a same time-different place configuration. The aviation maintenance person views the rotor via digital video on their office workstation while the logistics supervisor concurrently views the rotor through their HMD. The aviation maintenance person indicates where to look via drawing augmented reality lines over the video image. These augmented reality line drawings are registered to the rotor's container. Locale tracking infrastructure such as fiducial markers or radio beacons may be placed on the container to improve such registration. The aviation maintenance person then directs the logistics supervisor to read information on an indicated information plate. The aviation maintenance person can show digital images of similar rotors or they can show a 3D model, for example, to highlight a particular location on the rotor. Both parties may make use of augmented reality information added to the other persons view to improve communication

Once the two people agree this is the correct rotor the logistics supervisor places a virtual note on the rotor's container indicating it is an urgent request and has the rotor placed on the plane for shipping. The virtual note is stored in a logistics information system and is retrieved by a query of the identification of the smart badge attached to the container. This becomes a different time-different place configuration, as the airstrip clerk the second airfield will read this note at a later date in a different location. Once the plane lands in the other country, the airstrip clerk reads the augmented reality information sticker and expedites the rotor to the helicopter base. The rotor is then placed on a truck with other required items to be sent to the location of the helicopter. While in transit, the truck runs off the road, falls on its side, and dumps its contents onto the side of the road. Many of the containers are damaged. The aviation maintenance person from the helicopter base is contacted and drives out to the site to inspect the rotor and other items. While at the crash site, the aviation maintenance person and truck driver inspect the different containers for damage and reviews each of the manifests as augmented reality information. This becomes a same time-same place configuration. The rotor's container 
has the urgent virtual information tag on it, and the aviation maintenance person inspects this container first. They find the rotor to be damaged, and the aviation maintenance person contacts the logistics supervisor to get a new rotor ordered. The other items such as clothing are sent on a new truck to the helicopter base.

A key difference with this form of collaboration is the artefact the users are manipulating. This artefact can be characterised by the following features: firstly, it corresponds to the physical world; secondly, the size of the information space reflects the physical objects in a large area; and thirdly, the users are able to physically walk within the information space and the physical world simultaneously. This form of collaboration is similar to distributive virtual environment collaboration systems. Both have manipulable 3D models and the position of the users affects their vantage point. The significant differences are that the distances the users are allowed to physically move are larger, and there is a one-to-one correspondence with the physical world.

\section{The Original Tinmith-Hand}

Current augmented and virtual reality systems by in large are oriented toward information presentation: the user wearing a HMD, moving around the world, and experiencing the artificial reality. Tinmith-Hand builds on concepts from a number of VR interaction researchers, including: proprioception and the placement of objects relative to the body in Mine et al. [18]; the viewing and manipulations of objects using the Worlds-in-Miniature [19] and Voodoo Dolls [20] techniques; two-handed 3D interaction techniques [21,22]; selection and manipulation techniques like the GoGo arm [23] and various others covered in Bowman and Hodges [24].

The main interactions with Tinmith-Hand [25] are through head and hand gestures (although voice recognition is another viable option), and so we wish to keep the hands free from holding input devices if possible. The primary user interaction for graphical object manipulation will be through the 3D tracking of the user's head and two electronic pinch gloves. The gloves operating in a pinch mode will control the menu system. The goal of the menu system is to allow the user to easily access the functionality of the application, in a logical manner, while not obscuring the user's vision or preventing other tasks.
The user operates an application with the TinmithHand user interface, using head movement, hand tracking, pinch gloves, and a menu system to perform the following object manipulation tasks:

- Object selection: the user can point at objects and select them, placing them into one of several clipboards.

- Object transform: perform translate, rotate, and scale operations, in a variety of different ways.

- Create primitives: 3D primitives can be created in the virtual world, from infinite planes as the most primitive, to complex graphical models such as a water heater or helicopter rotor.

- Combine primitives: previously constructed and manipulated primitives may be combined together using Constructive Solid Geometry (CSG) operations to produce higher level graphical objects.

The following components are used to implement the user interface and applications, used to construct large graphical objects outdoors:

- Menu system and pinch gloves: the command interface to the system through the pinch action of our gloves. These gloves were custom built to integrate in with the rest of the system.

- Four integrated pointing techniques: the system is capable of using four interchangeable pointing devices to supply input, depending on the requirements at the time and the suitability. The devices are one and two-handed thumb tracking, a head orientation eye cursor, and a track ball mouse.

- Image plane interaction techniques: these techniques are where the objects are manipulated on a 2D plane perpendicular to the current view direction [26]. By combining pointing with image plane techniques, it is possible to manipulate objects in a 3D environment, moving the camera angle simply by walking.

- Application tailored menus: to support the domain specific construction application, menu options are added that tailor the menu system to the domain specific tasks.

- CSG operations: users intuitively understand operations such as carving and combining objects. We have leveraged this understanding by basing the interactive construction of complex real world shapes around the use of CSG operations. 


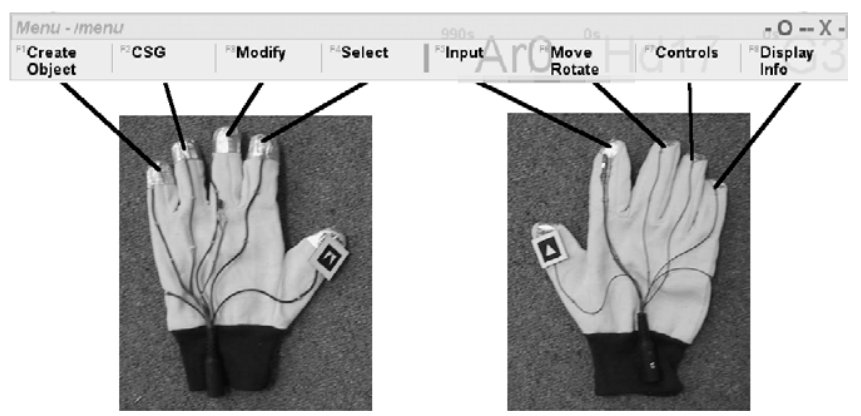

Fig. 3. Root menu node layout, with corresponding finger mappings.

\section{Menuing System}

The menuing system of Tinmith-Hand provides the ability to trigger commands without the use of a keyboard or traditional mouse. We constructed a set of pinch gloves, similar to the Pinch Gloves produced by FakeSpace [27], to drive the menu system in a handsfree manner. Our gloves send signals to the computer indicating which fingers are currently pressed into either the palm or the thumb, and when the appropriate finger is pressed, the menu node is selected. The menu options (Fig. 3) are presented in a transparent green dialog box at the bottom of the display, which can be repositioned if desired. We used transparency to avoid visual clutter caused by menu boxes, by allowing the user to see through the menu. The menu colours and transparency are dynamically changeable.

Each menu option is assigned to a finger on the gloves. To select an option, the user touches the matching fingertip with the thumb tip. For example the Modify option would be selected if the middle finger and thumb of the left hand were pressed together. To indicate a selection, the user must hold the press for a short period of time to eliminate key bounce problems or accidental brushing of the glove. When the press is complete, the system generates a beep and then moves to the selected node in the menu hierarchy. The system then can execute an action at this node if required. In addition, Tinmith-Hand may present a new set of options or return back to the top level of the menu structure if the operation is complete. By pressing any finger to the palm of the glove, the menu returns back to the top level.

A menuing system developed at a similar time as ours is an immersive VR system using Pinch Gloves [27], recently described in Bowman and Wingrave [28]. Although a similar concept as ours, it was different in that it was very much like traditional pull down menus. The top-level menu items were available on one hand, and the second level options on the other hand. Using the small finger it was possible to cycle through options if there were more than three options. The menus were limited to a depth of two, and it is not scaleable to a large number of hierarchical commands. Our system is fundamentally different due to the way the user interacts with the menu. Our menus do not float in the 3D world like other VR menus $[18,28]$, since we feel that these menu options should always be visible during the operation of an application. The menus may be removed as the user desire, through options under the user's control.

\section{Gloves and Gesture Interfaces}

Tinmith-Hand is also designed to support applications that interact with graphical objects and enter spatial information. We chose hand gestures to be the main interaction method for the user interface. To track the location of the gloves, small fiducial markers are placed on the thumbs, and a camera mounted on the HMD feeds live video into the laptop. The ARtoolkit software library [29] is used to process the image to recover a $3 \mathrm{D}$ transformation matrix relative to the camera, allowing us to calculate the position of the user's hands in world coordinates.

Given the location of the hands, the system overlays registered 3D axes. At the same time, a 2D flat cursor is overlaid on top. The cursor is placed in a desired location by movement of the user's hand. When the user activates selection using the menu and gloves, a ray is fired into the scene from the 2D cursor, and the first object hit is selected. When a user performs a pick operation on a graphical object, the system determines the closest polygon under the cursor. When a polygon is selected, the simplest object is chosen, but the user can traverse up the scene graph to select more of the model if desired. Every polygon and object in the scene exists in the world model hierarchy; many objects are also children of other objects. 


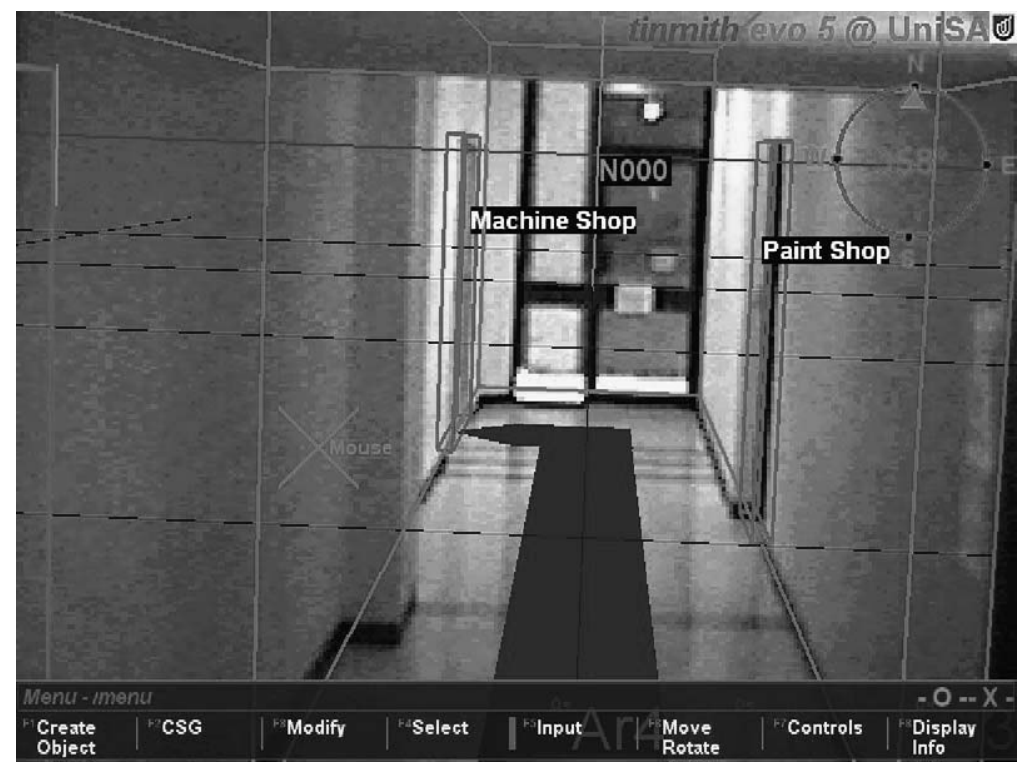

Fig. 4. Augmented reality navigation cues.

\section{The New Tinmith-Hand to Support AR Information}

We have extended our user interface system TinmithHand $[25,30]$ that combines the tracking of the gloves and a menu system to from a complete user interface solution that is capable of controlling an OWARCS application. We have developed a set of augmented reality information presentation techniques based on the previously presented logistics scenario. The three selection techniques we have implemented are presented next, two-handed framing, line of sight and laser beam. Finally, a new glove based text entry mechanism we have implemented is discussed.

\section{Presentation of Augmented Reality Information Stickers}

We believe the use of hand and head gestures are key to making outdoor augmented reality usable. This section presents a number of augmented reality user interface mechanisms to support the OWARCS presented in the previous logistics scenario. These augmented reality user interface mechanisms will be presented in the same order as presented in the logistics scenario.

The aviation maintenance person places an urgent request for the replacement in a workflow system via a traditional workstation. The workflow system coordinates with the logistics inventory system and specifies a particular rotor to be shipped. The new Tinmith-Hand provides navigation cues to the user to retrieve this rotor in the warehouse, by the use of virtual signposts, virtual line markings on the floor, and augmented reality information stickers. Figure 4 is the user's view of two different navigation cues. We are currently investigating an indoor tracking system to support such visualisations. Our current system works with traditional tracking devices, such as a Polhemus tracker and GPS. These augmented reality information stickers function similar to the situation sensitive information described in Rekimoto et al. [31,32]. There are three virtual signposts in the user's view in the figure, 'Machine Shop', 'Paint Shop' and 'N000'. The 'Machine Shop' and 'Paint Shop' virtual signposts indicate entrances to those facilities. The 'N000' virtual signpost indicates a compass heading of due north; there are virtual signposts for the eight points of the compass. On the floor there is a red arrow headed thick line providing a virtual walking path for the user. (The greyscale images obviously do not reflect the red.) This virtual information is automatically generated. The virtual signposts are automatically placed in the user's view once the new Tinmith-Hand system initiates the requirement for the user to retrieve the rotor. At the same time, the new Tinmith-Hand generates a set of virtual line markers showing how to walk to the desired item.

A fourth navigation cue is a top down map, providing the user with a gods-eye view of the world, looking down onto the Earth. As the user rotates their head, 


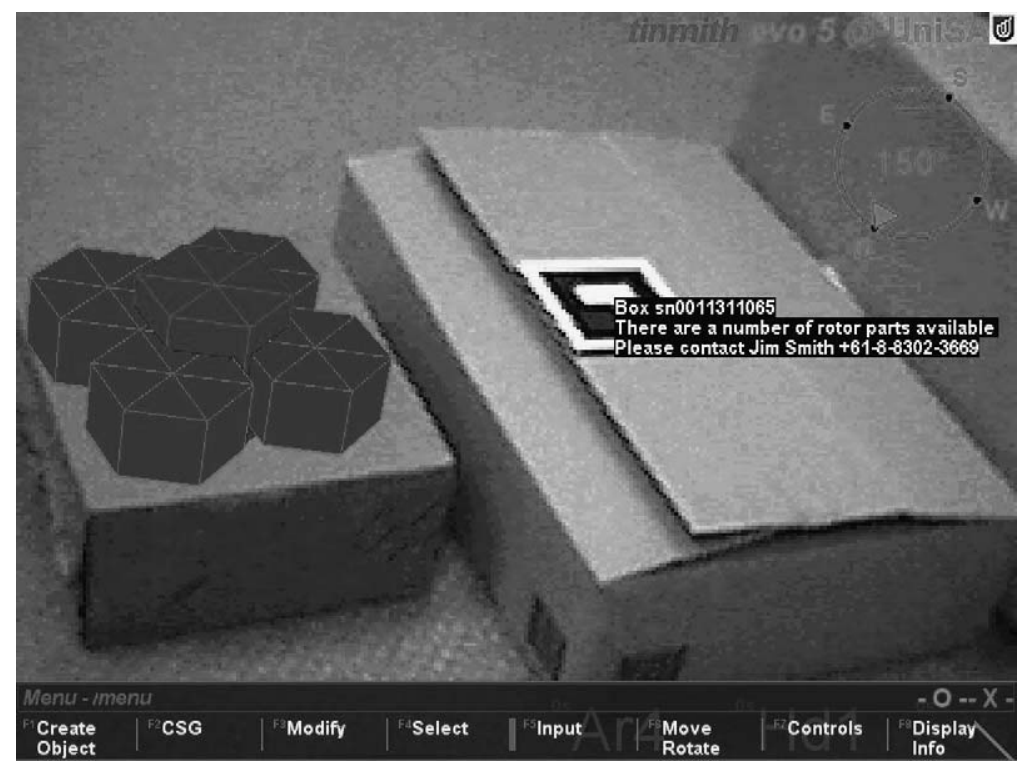

Fig. 5. Multimedia augmented reality sticker.

the entire map rotates as well, with the user's direction being toward the top of the display.

The new Tinmith-Hand supports a number of different forms of information stickers, such as: text, line drawings, and 3D graphical objects. In the future we will be adding new media types, such as audio, voice, digital images and digital video. The augmented reality information stickers must be designed to be viewed from a number of directions and distances. Figure 5 shows two different forms of multimedia augmented reality information stickers. There is text label 'Box sn00 ...' attached to the container in the right side of the diagram. The container on the left side of the diagram has a 3D graphical model depicting the helicopter rotor. This information may be placed in world coordinates (as is the instance in Fig. 5) or in screen coordinates (as is the case in Fig. 7). In the case of viewing 3D graphical objects, we support direct manipulation of the objects as well as a second useful camera control model, orbital mode, as described in [33].

Figure 6 shows the workstation view from the remote aviation maintenance person's perspective. The lower right window on the aviation maintenance person's display is the same view as the logistics supervisor. In the future we will add the ability for the aviation maintenance person and the logistics supervisor to annotate their views. The aviation maintenance person, for example, will be able to highlight regions of interest to draw the attention of the logistics supervisor. This highlight may be either screen or world relative. The upper right window is a detailed 3D model of the object in question, and the upper left window is a text description of the object depicted in the 3D model.

At the site of the crash in the scenario, the aviation maintenance person and truck driver view the manifests for each of the containers as screen relative text boxes, as shown in Fig. 7. As a container comes into the user's view, an augmented reality text label is attached to the container. A screen relative text box depicts the different manifests for each of the containers currently in the view of the user. A total manifest for the contents of the truck may also be viewed in a text box with appropriate scrolling and paging.

In the helicopter rotor example, both users were required to indicate features in the physical world or on the 3D models. Hand and head gestures are an intuitive means for indicating features in the physical world or on the 3D models. For example, one user may wish to indicate a particular feature by framing the region with their two thumbs (Fig. 8), or line of sight to the tip of their thumb (Fig. 9), or a laser beam from the tip of their thumb (Fig. 10). Our two hand framing and line of sight techniques are implemented as extensions to image plane interaction techniques. The laser beam technique is a full six degree of freedom selection technique. We have implemented a traditional fixed length laser beam/ray casting selection device. Once a region or object is selected, this is then highlighted on the desktop display and/or the HMD to provide additional visual cues to the user. For the HMD users, control of the selected region could be transferred to the headtracking device for gross movements and the hands (using a magic lens interaction technique for example) 


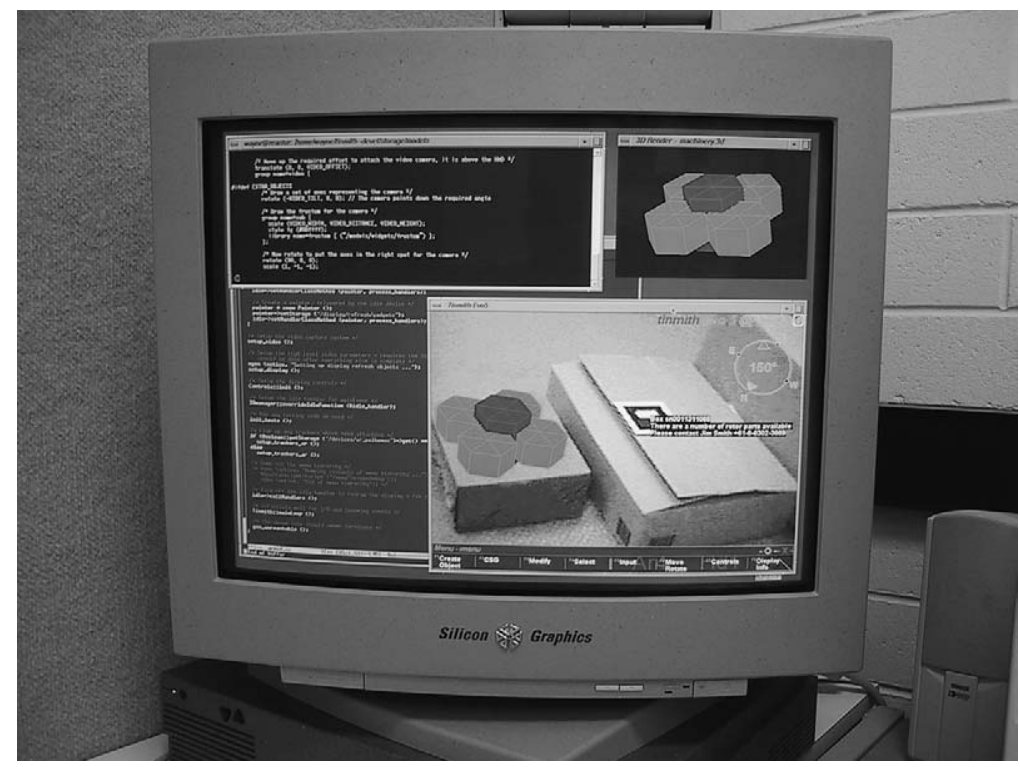

Fig. 6. Screen shot of the workstation with annotations.

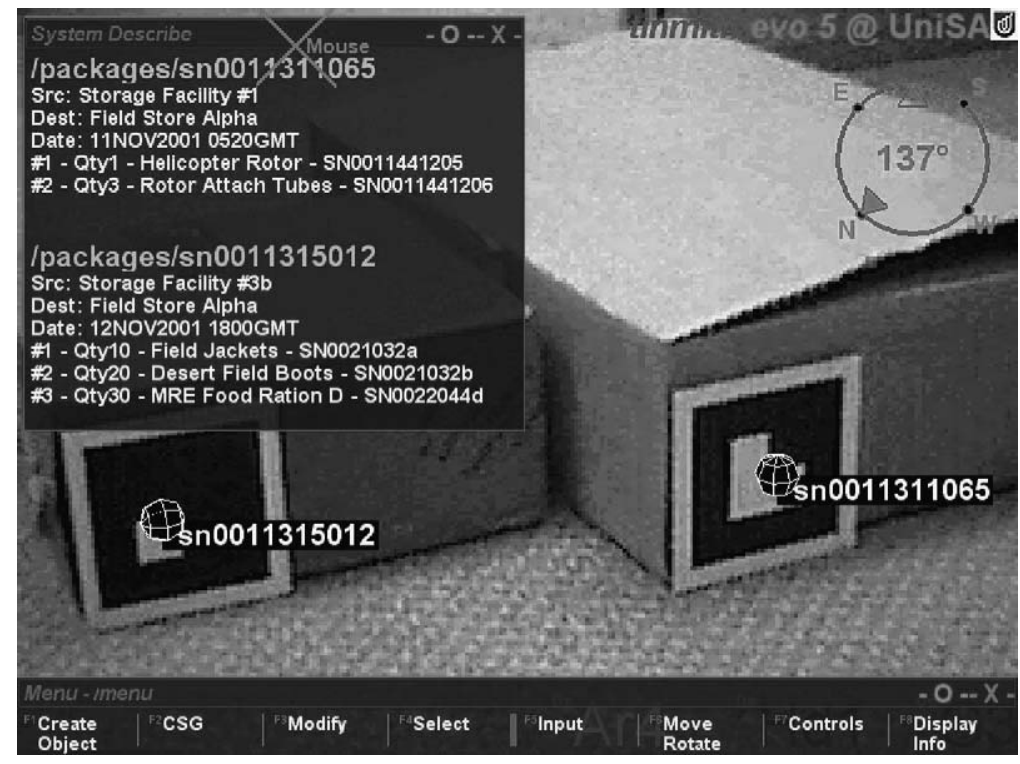

Fig. 7. Manifests of each of the containers.

may perform finer control. We believe the ability to quickly change input devices and coordinate systems is a key to making this form of interaction feasible.

\section{Two-handed Framing}

Two-handed framing is performed by initiating the selection process through the activation of the correct menu control. Once the selection task has begun, a 2D yellow frame appears between the user's thumbs. This rectangular frame is orientated relative the user's screen. Two-handed rubber banding can then be used to frame an area or object of interest [34]. As with traditional two-handed rubber banding, the control of two opposite corners of the rectangular region allows the user to simultaneously control the size and placement of the region. Once the desired region has been chosen, the selection is finalised with a pinch to the correct menu item. A limitation of using the vision based tracking is both hands are required to be in view of the camera at all times. In our configuration, the field 


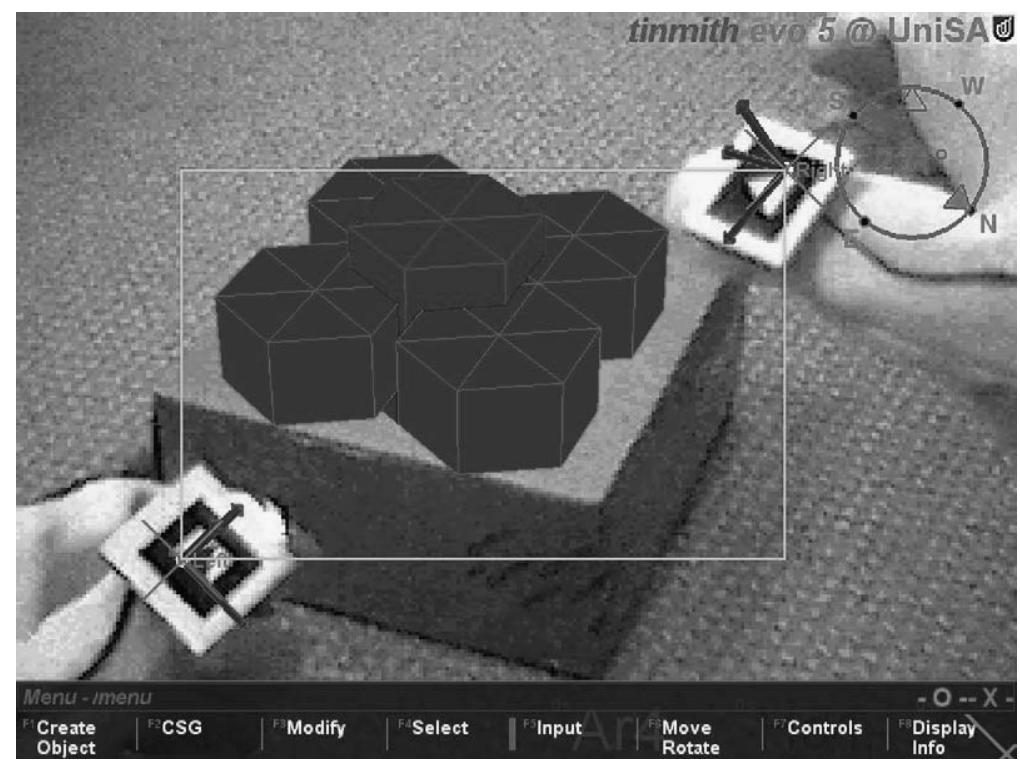

Fig. 8. Two-handed framing.

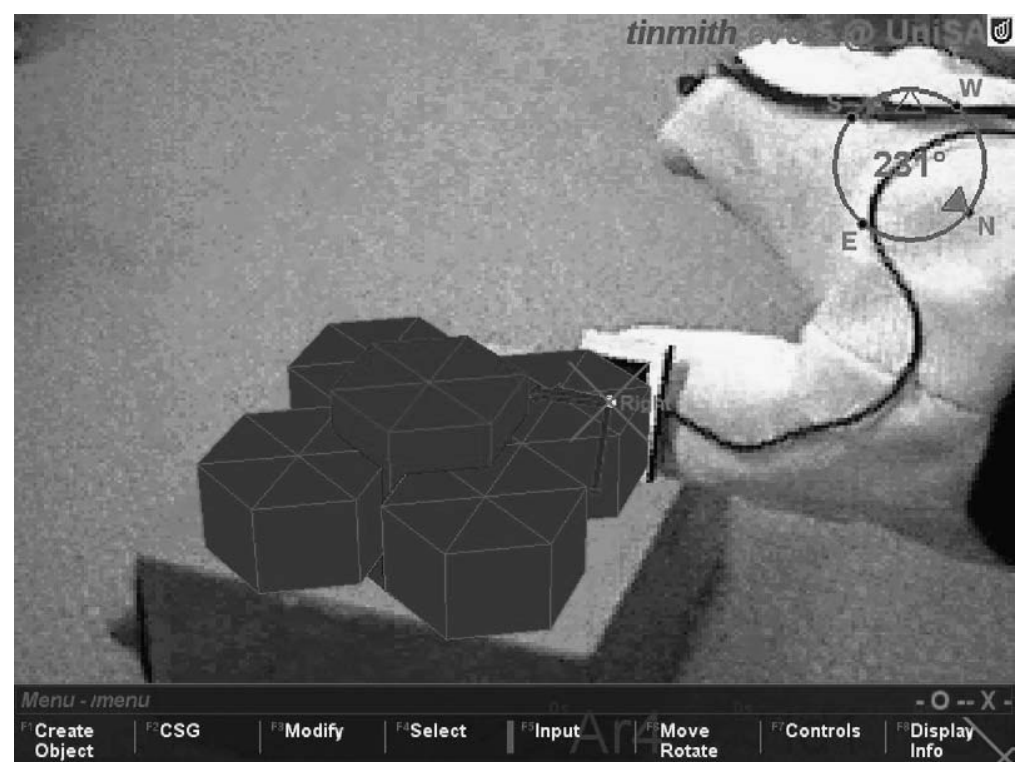

Fig. 9. Line of sight.

of view of the camera is larger than the field of view of HMD, and the image is properly distorted to fit within the HMD using the scene graph and avatar model.

\section{Line of Sight}

As previously mentioned, the line of sight selection method is also an image plane interaction technique, but line of sight only selects a small area region. In our example the defined region is a small cone in the immediate region of the thumb fiducial marker. Figure 9 depicts how the user would select the 3D graphical model by forming a virtual line from the user's eye through the centre of the thumb fiducial marker, and that line intersecting with the graphical model. (These figures were captured using the system in the video see-through mode, and as such provides model occlusion with the physical world.) The selection is activated with a glove pinch on the other hand; in this case that would be the left hand. 


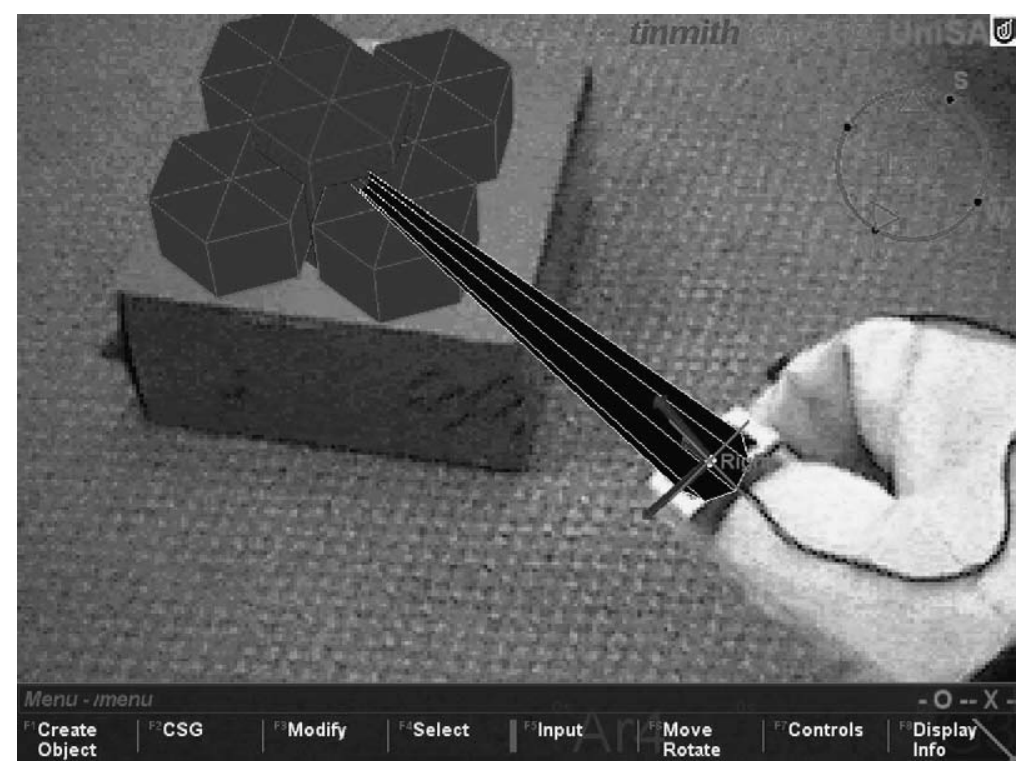

Fig. 10. Laser beam,

\section{Laser Beam}

The laser beam technique uses a traditional virtual reality laser beam/ray casting selection cursor. The length of the laser beam is fixed, and this example it is set to two meters. This technique is functionally quite similar to the line of sight technique. In our laser beam example, the line is a cone and the direction and location is specified with the six degree of freedom tracking of the user's thumb. This technique has greater sensitivity to tracker noise from the ARToolkit than the others. The size of the target, changing lighting conditions, and slow tracker camera update rates all contribute to the level of noise. In addition, the nature of a laser beam acting as a long lever accentuates angular deviation of the tracker.

\section{Entering Text}

The previously mentioned scenario requires the user to enter short text messages. We envision these messages to be of similar length to mobile phone SMS messages, which allow a maximum of 160 character length messages. These messages use a subset of the total ASCll character set, and incorporate a range of abbreviations and a specialised language; for example the use of ' $r$ ' instead of are and ' $c$ u I8r' for see you later [35]. The design of the text entry mechanism is centred on our current pinch glove input device; we did not wish to add an extra input device. The second design feature is a constant online help feature. When a user activates a text entry dialog box, the user is presented with the entire key mappings for user finger presses with pinch gloves and a text entry field.

Figure 11 depicts the layout for the keying combination for 49 characters of the simultaneous pressing of one or two fingers on the thumb for both of the hands. The grid mapping in the figure is designed for the user's hand posture assuming the right hand in an orientation of the fingers pointing down - palm facing the user and the left hand fingers pointing to the right also with the palm facing the user. Each column in the matrix is mapped from left to right as the following right hand finger combinations with the thumb: (1) index, (2) index and middle, (3) middle, (4) middle and ring, (5) ring, (6) ring and pinkie, and (7) pinkie. The same mappings are used for each row using the left hand. For example pinching the thumb and index finger (RF1) of the right simultaneously while pinching the thumb and middle (LF2) and ring finger (LF3) of the left hand would enter the ' $\mathrm{V}$ ' character. A simple delete editing command is provided with the touching of any finger on the left hand to its palm; the touching of any finger on the right hand to its palm ends the text entry mode. The key mappings may be changed for different orientations of the hands, say the right hand having the fingers pointing up. The optimal configuration is an area to be investigated.

Glove input devices, such as the 'chording glove' [36] have been used as devices to emulate five button chording devices, one button for each finger on a hand. 


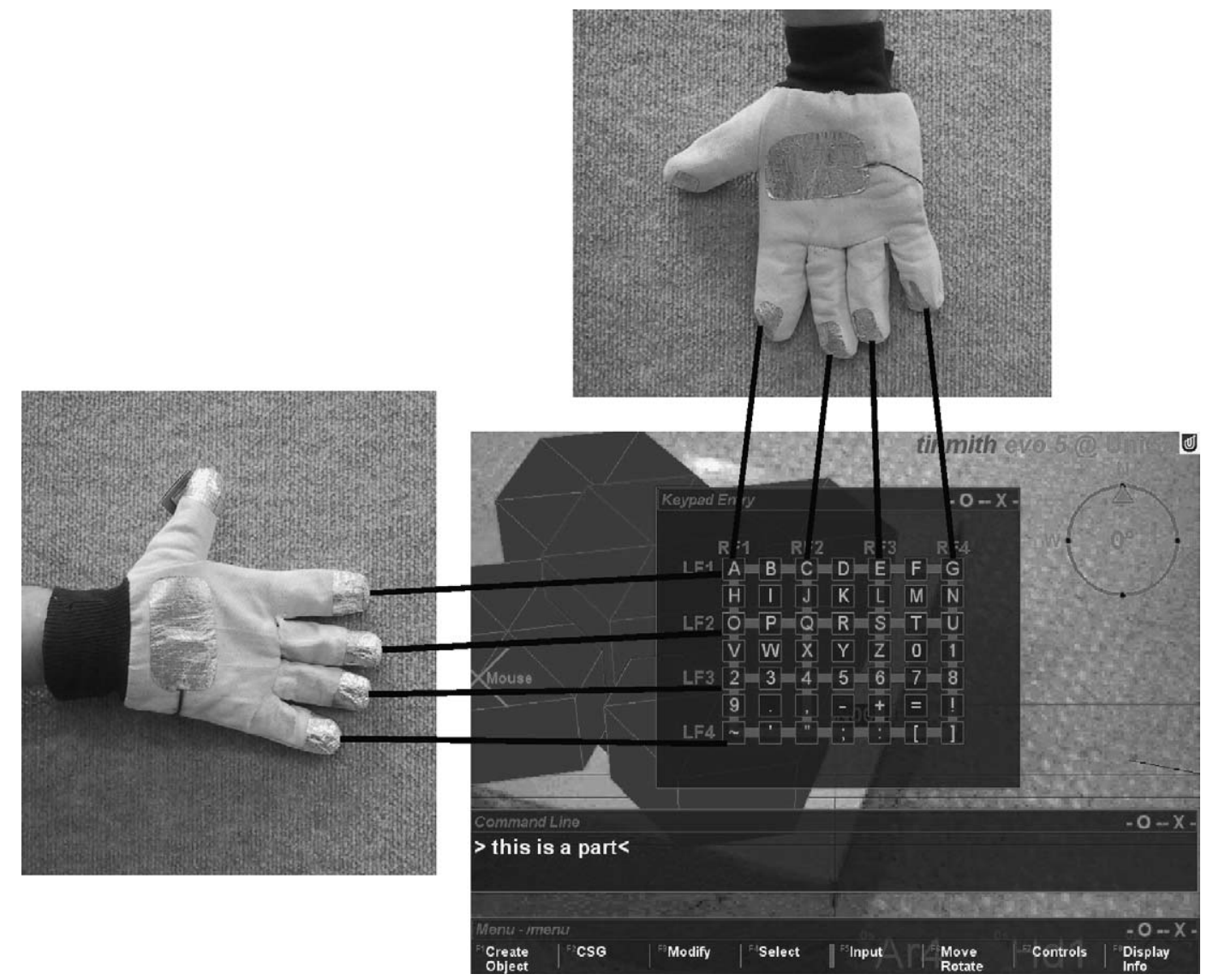

Fig. 11. Initial mapping of letters to particular fingers. (The view of the hands is of the palms, and the naming of fingers are as follows: index, middle, ring, and pinkie.)

One main difference with our approach is we do not attempt to replicate the entire OWERTY keyboard. As we are supporting only short text messages, an easier and simpler approach is more appropriate requiring no training. The chording glove and other hand pose chording techniques may impact on the ability of users to perform other tasks with their hands, such as lifting boxes and operating machinery. Physical buttons have been placed on the fingertips of the chording glove, which can be accidentally activated and they reduce the dexterity of the fingers.

\section{Tinmith System}

Our current OWARCS is implemented with the Tinmith evolution 5 system [37]. The Tinmith system is built up of both hardware and software, using off the shelf products and custom built components for our research, as some of our needs cannot be met with existing technology.

\section{Hardware}

The wearable computer system as shown in Fig. 1 is based on a Gateway Solo P2-450 laptop (64 mb RAM, ATI Rage OpenGL) mounted on a hiking backpack. An Intersense IS-300 hybrid tracker performs orientation sensing. Position information is gained from a Trimble Ag132 GPS with an accuracy of less than or equal to $0.5 \mathrm{~m}$. The display is a Sony Glasstron PLM700 e monocular SVGA display. A large $12 \mathrm{~V}$ battery powers the various trackers, as well as the small LCD television on the back for debugging and spectators to view. A SuperCam WonderEye USB video camera is used to provide images for the hand tracking system. The laptop runs RedHat Linux 7.0 with kernel 2.4 as its operating system, including the standard GNU 
development environment. XFree86 v3.3.6 is used for graphics, as it performs hardware accelerated OpenGL using Utah-GLX.

\section{Pinch Gloves}

As previously mentioned, we developed a pair of pinch gloves (shown in Fig. 3) as our main input device, which is similar to a number of existing technologies. The FakeSpace PinchGlove [27] contains electrical sensors at each fingertip to measure touching, while the VTi CyberGlove [38] uses bend sensors to measure finger positions. For our application, we require the ability to record finger touches, and so the Pinch Glove would be the most suitable. However, it has limitations in that only the fingertips are monitored, and we desired to have more functionality. The CyberGlove is designed for human motion capture and not pinch gestures, and so is not suitable for this task. As a result, a glove was constructed in-house to meet the criteria required.

Our glove is based around a typical gardening glove that loosely fits the hand, allowing easy removal. Special metallic tape (normally used to tape reflective insulation to inside house roofs) was adhered to the fingertips, thumb, and palm of the glove to provide a metallic surface. Wires were attached and run to the processing unit. We constructed a processing unit to interface with the laptop computer, which takes the load off the laptop for monitoring the glove gestures. A Parallax [39] Basic Stamp BS2 microcontroller was used, which is very easy to program, contains $16 \mathrm{I} / \mathrm{O}$ pins, a serial port, and is fully implemented on a single IC sized circuit

The gloves allow us to alter the pad location and size, and due to the palm pads, support other gestures apart from just pinching. The glove allows multiple fingers to be pressed, and detect the location of each finger individually. Gloves that use pressure sensors can only detect pressure, not where the pressure is applied, and gloves that use switches can detect contact but not against what surface.

\section{Conclusion}

In conclusion, this paper presents a set of augmented reality user interface technologies for an outdoor wearable augmented reality computer system to support activities such as collaboration. Hand and head gestures are the main form of user interaction. To highlight the effectiveness of such a collaboration system, a scenario of delivering a replacement helicopter rotor was described.
We extended our original user interface system Tinmith-Hand, which combines the tracking of the gloves and a menu system. The extension included a set of augmented reality information presentation techniques, which was developed based on the presented logistics scenario. The following three selection techniques were also developed: two-handed framing, line of sight, and laser beam. Finally, a new glove based text entry mechanism was implemented. To make the user interface technologies relevant, an overall system architecture for outdoor wearable augmented reality computer system (OWARCS) was presented.

\section{Acknowledgements}

The authors would like to especially acknowledge the work of Arron and Spishek Piekarski, who both helped in the construction and design of the glove and HMD. Thanks also to the Division of ITEE and Defence Science Technology Organisation (DSTO).

\section{References}

1. Azuma RT (1997) Survey of augmented reality. Presence: Teleoperators and Virtual Environments 6

2. Hollerer T, Feiner S, Terauchi T, Rashid G, Hallaway D (1999) Exploring MARS: developing indoor and outdoor user interfaces to a mobile augmented reality system. Computers and Graphics 23: 779-785

3. Feiner S, Maclntyre B, Hollerer T, Webster A (1997) A touring machine: prototyping 3D mobile augmented reality systems for exploring the urban environment. In: International Symposium on Wearable Computers: IEEE, 74-81

4. Julier S, Baillot Y, Lanzagorta M, Brown D, Rosenblum L (2000) Bars: Battlefield augmented reality system. In NATO Symposium on Information Processing Techniques for Military Systems, Istanbul, Turkey

5. Piekarski W, Thomas BH, Hepworth D, Gunther B, Demczuk V (1999) An architecture for outdoor wearable computers to support augmented reality and multimedia applications. In: Proc 3rd International Conference on Knowledge-Based Intelligent Information Engineering Systems. Adelaide

6. Thomas BH, Demczuk V, Piekarski W, Hepworth D, Gunther B (1998) A wearable computer system with augmented reality to support terrestrial navigation. In: Second International Symposium on Wearable Computers, Pittsburgh

7. Thomas B, Close B, Donoghue J, Squires J, DeBondi P, Morris M, Piekarski W (2000) ARQuake: An outdoor/ indoor augmented reality first person application. In: 4th International Symposium on Wearable Computers, Atlanta, GA

8. Azuma RT (1999) The challenge of making augmented reality work outdoors. In: 1 st International Symposium on Mixed Reality (ISMR '99), Yokohama, Japan

9. TeamBoard Inc. (2001) TeamBoard 300 Hanlan Road, Woodbridge, Ontario, Canada L4L 3P6 
10. Fagrell H, Forsberg K, Sanneblad J (2000) Fieldwise: a mobile knowledge management architecture. In: Conference on Computer Supported Cooperative Work, Philadelphia

11. Thomas B, Grimmer K, Makovec D, Zucco J, Gunther B (1999) Determination of placement of a body-attached mouse as a pointing input device for wearable computers. In: International Symposium on Wearable Computers, San Francisco, CA

12. Reitmayr G, Schmalstieg D (2001) Mobile Collaborative Augmented Reality, presented at 2nd ACM/IEEE International Symposium on Augmented Reality (ISAR'01), New York, NY

13. Höllerer T, Feiner S, Hallaway D, Bell B, Lanzagorta M, Brown D, Julier S, Baillot Y, Rosenblum L (2001) User interface management techniques for collaborative mobile augmented reality. Comput \& Graph 25: 799-810

14. Bauer M, Kortuem G, Segall Z (1999) 'Where are you pointing at?' A study of remote collaboration in a wearable videoconference system. In: International Symposium on Wearable Computers, San Francisco, CA

15. Billinghurst M, Bowskill J, Jessop M, Morphett J (1998) A wearable spatial conferencing space. In: 2nd International Symposium on Wearable Computers, Pittsburgh

16. Thomas BH (2001) Using augmented reality to support collaboration in an outdoor environmen. In: Special Session Augmented Reality: Usability and Collaborative Work in the $\mathrm{HCl}$ International, New Orleans, LA

17. Ellis C, Gibbs S, Rein G (1991) Groupware - some issues and experiences. Commun ACM 34: 9-28

18. Mine M, Brooks FB, Sequin CH (1997) Moving objects in space: exploiting proprioception in virtual-environment interaction. In: SIGGRAPH, Los Angeles, CA

19. Stoakley R, Conway MJ, Pausch R (1995) Virtual reality on a WIM: interactive worlds in miniature. In: Conference on Human Factors in Computing Systems, Denver, CO

20. Pierce JS, Steams BC, Pausch R (1999) Voodoo Dolls: seamless interaction at multiple scales in virtual environments. In: Symposium on Interactive 3D Graphics, Atlanta, GA

21. Hinckley K, Pausch R, Goble JC, Kassell NF (1994) A survey of design issues in spatial input. In: 7th Int Symposium on User Interface Software Technology, Marina del Rey, CA

22. Zeleznik RC, Forsberg AS, Strauss PS (1997) Two pointer input for 3D interaction. In: Symposium on Interactive 3D Graphics, Providence, RI

23. Poupyrev I, Billinghurst M, Weghorst S, Ichikawa T (1996) The Go-Go interaction technique: non-linear mapping for direct manipulation in VR. In: 9th Int Symposium on User Interface Software Technology, Seattle, WA

24. Bowman DA, Hodges LF (1997) An evaluation of techniques for grabbing and manipulating remote objects in immersive virtual environments. In: Symposium on Interactive 3D Graphics, Providence, RI

25. Piekarski W, Thomas BH (2001) Tinmith-Metro: new outdoor techniques for creating city models with an aug- mented reality wearable computer. In: 5th International Symposium on Wearable Computers, Zurich, Switzerland

26. Pierce J, Forsberg A, Conway M, Hong RZ, Mine M (1997) Image plane interaction techniques in 3D immersive environments. In: Symposium on Interactive 3D Graphics, Providence, RI

27. FakeSpace Labs, Pinch Gloves (Last accessed: Oct. 30, 2001). www.fakespacelabs.com/products/pinch.html

28. Bowman DA, Wingrave CA (2001) Design and evaluation of menu systems for immersive virtual environments. In Virtual Reality, Yokohama, Japan

29. Kato H, Billinghurst M (1999) Marker tracking and HMD calibration for a video-based augmented reality conferencing system. In: Proc 2 nd IEEE and ACM International Workshop on Augmented Reality '99, San Francisco, CA, 85-94

30. Piekarski W, Thomas B (2002) The Tinmith System demonstrating new techniques for mobile augmented reality modelling. In: Australasian User Interface Conference, Melbourne

31. Rekimoto J, Najao K (1995) The world through the computer: computer augmented interaction with the real world. In: User Interface Software and Technology, Pittsburgh

32. Rekimoto J, Ayatsuka Y, Hayashi K (1998) Augmentable reality: situated communication through physical and digital spaces. In: 2nd International Symposium on Wearable Computers, Pittsburgh

33. Koller DR, Mine MR, Hudson SE (1996) Head-tracked orbital viewing: an interaction technique for immersive virtual environments. In: 9th Int Symposium on User Interface Software Technology, Seattle, WA

34. Buxton W, Myers B (1986) A study in two-handed input. In: Conference on Human Factors and Computing Systems

35. Butts L, Cockburn A (2002) An evaluation of mobile phone text input methods. In: 3rd Australasian User Interface Conference, Melbourne

36. Rosenberg R, Slater M (1999) The chording glove: a glove-based text input device. IEEE Trans Syst, Man and Cybernetics, Part C: Applic and Rev 29: 186-191

37. Piekarski W, Thomas B (2001) Tinmith-evo5: an architecture for supporting mobile augmented reality environments. In: 2nd IEEE and ACM International Symposium on Augmented Reality, New York, NY

38. Virtual Technologies, CyberGlove www.virtex.com/ products/hw-products/cyberglove.html

39. Parallax, Basic Stamp BS2 (last accessed: Nov. 14 2001) www.parallaxinc.com

Correspondence and offprint requests to: $B . H$. Thomas, Wearable Computer Laboratory, School of Computer \& Information Science. University of South Australia, Mawson Lakes, SA 5095. Australia. http://wearables. unisa.edu.au 